\title{
Infrahepatic Inferior Vena Cava Clamping does not Increase the Risk of Pulmonary Embolism Following Hepatic Resection
}

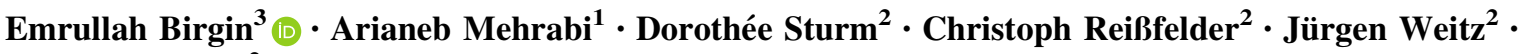 \\ Nuh N. Rahbari ${ }^{2}$
}

\begin{abstract}
Background Infrahepatic inferior vena cava (IVC) clamping reduces central venous pressure. However, controversies remain regarding its impact on postoperative complications, particularly, the incidence of postoperative pulmonary embolism (PE). The aim of the study was to determine the impact of IVC clamping on the incidence of $\mathrm{PE}$ in patients undergoing hepatectomy.

Methods A pooled analysis of five prospective trials on patients who underwent hepatic resection over a period of 10 years was performed. Patients with infrahepatic IVC clamping were compared to patients without infrahepatic IVC clamping. Outcomes were studied by univariate and multivariate analyses.

Results Of 505 included patients, 141 patients had IVC clamping and 364 patients served as control group. The rate of postoperative PE was comparable between groups (3\% vs. $3 \% ; P=0.762)$, as were postoperative morbidity $(P=0.932)$, bile leakage $(P=0.272)$, posthepatectomy hemorrhage $(P=0.095)$, and posthepatectomy liver failure $(P=0.605)$, respectively. No clinicopathological and intraoperative risk factors were found to predict the onset of PE. Subgroup analyses of patients with major hepatectomy and vascular resections confirmed no adverse perioperative outcomes to be associated with IVC clamping.

Conclusions Infrahepatic IVC clamping does not increase the incidence of postoperative PE.
\end{abstract}

Nuh N. Rahbari

nuh.rahbari@umm.de

1 Department of General, Visceral and Transplantation Surgery, University of Heidelberg, Heidelberg, Germany

2 Department of Gastrointestinal, Thoracic and Vascular Surgery, University Hospital Carl Gustav Carus at the Technische Universität Dresden, Dresden, Germany

3 Present Address: Department of Surgery, Medical Faculty Mannheim, University Medical Center Mannheim, Heidelberg University, Theodor-Kutzer-Ufer 1-3, 68167 Mannheim, Germany

\section{Introduction}

Hepatic resection is the treatment of choice for benign and malignant liver tumors [1, 2]. Despite substantial improvements in perioperative mortality within the past three decades, morbidity after hepatic resection remains high with rates up to $60 \%$ [3]. There is a strong association of postoperative complications with the amount of intraoperative blood loss [4]. Thus, various intraoperative strategies to limit blood loss were developed including low central venous pressure (CVP) during hepatic transection and vascular occlusion techniques $[5,6]$. Infrahepatic clamping of the inferior vena cava (IVC) is a method of outflow control to maintain a low CVP. Compared to the commonly applied anesthesiological approach to lower CVP by fluid restriction, IVC clamping can be used in 
euvolemic patients with the advantage of less hemodynamic instability and improved organ perfusion [7]. In a previous randomized controlled trial, we found IVC clamping to be effective in lowering CVP and reducing intraoperative blood loss. However, it was also associated with a significantly higher incidence of postoperative pulmonary embolism (PE) [8]. Indeed, several other randomized controlled trials did not show any morbidity related to the use of infrahepatic IVC clamping during hepatectomy, though heterogeneous surgical techniques were applied with respect to routine use of inflow control and the extent of IVC clamping [9-12]. Furthermore, two recent meta-analyses of randomized controlled trials, mainly involving cohorts with primary liver malignancies, suggested no adverse events following IVC clamping within the above-mentioned limitations [13, 14].

Due to the heterogeneous data, it was the aim of the present analysis to assess the impact of IVC clamping on postoperative complications and in particular the incidence of PE in patients undergoing hepatic resection. To obtain highly valid and reliable data, we used individual patient data from registered and published trials that documented the use and duration of infrahepatic IVC clamping during elective liver resection in a prospective fashion.

\section{Materials and methods}

This study was performed as a secondary data analysis of four randomized controlled trials (NCT00732979, NCT01049607, NCT01858987, NCT02612220) and one prospective cohort study (NCT01073345) conducted between April 2007 and September 2017 at the Department of General, Visceral and Transplantation Surgery, University of Heidelberg, and the Department of Gastrointestinal, Thoracic and Vascular Surgery, University Hospital Carl Gustav Carus, Technische Universität Dresden $[8,15-18]$. These trials were selected due to the use of consistent surgical techniques in these institutions with or without the use of continuous (non-intermittent) IVC clamping during hepatectomy in primary and secondary liver malignancies. The included trials applied inflow control (Pringle maneuver) only in case of severe bleeding (and documented as secondary endpoint) while the perioperative care was identical in each case. Five other published trials on the use of IVC clamping were excluded due to (1) the application of IVC clamping in highly selective patient cohorts (e.g., with large hepatocellular carcinoma) [19], the restricted use of modified surgical hepatectomy techniques (e.g., anterior-approach) [11], (2) the concomitant use of Pringle maneuver with IVC clamping in all liver resections [9, 10], and (3) the use of a partial IVC clamping technique instead of a continuous IVC clamping technique [12]. The study was done in accordance with the Principles of Good Practice of Secondary Data Analysis (GPS). The present cohort study with secondary data analysis using de-identified data did not require an Institutional Review Board review according to our local institutional review policy.

\section{Patient eligibility criteria and data extraction}

Patient eligibility criteria for the individual trials were reported in the original publications. Patients were included in the present analysis in case hepatic resection was carried out and data on the use of infrahepatic IVC clamping were available. Patients who required infrahepatic IVC clamping for the purpose of IVC resection were excluded. The following data were extracted from the individual databases for the purpose of the present analysis: age, gender, body mass index (BMI), American Society of Anesthesiologists (ASA) score, diagnosis, presence of liver steatosis, presence of liver fibrosis, presence of liver cirrhosis, history of chemotherapy, history of hepatic resection, perioperative laboratory tests including bilirubin, aspartate aminotransferase, alanine aminotransferase, alkaline phosphatase (AP), gamma glutamyltransferase, creatinine, hemoglobin, platelets, and international normalized ratio (INR). In addition, the following operative details were extracted: extent and type of resection, number of resected segments, creation of bilioenteric anastomosis, and technique of hepatic parenchymal transection.

\section{Definitions and outcomes}

The primary endpoint was the incidence of postoperative PE. Postoperative PE was defined as pulmonary arterial obstruction and confirmed by spiral computed tomography of the chest in all suspected cases presenting with respiratory insufficiency [20]. Routine and scheduled computed tomography scans to rule out PE were not performed in the included studies [7, 8]. All patients with PE were transmitted to the intermediate/or intensive care unit and treated with therapeutic doses of low molecular weight or unfractionated heparin. Thromboprophylaxis was performed in the studies in line with the German guidelines on prophylaxis of thromboembolism which was initially published in 2003 [21]. In brief, all patients used elastic stockings and thromboprophylaxis with low molecular weight or unfractionated heparin irrespective of intraoperative vascular resections or type of surgery. The ClavienDindo classification was used to document the severity of postoperative complications. Clinically relevant complications were defined as Clavien-Dindo complications grade III and higher. Posthepatectomy bile leakage, posthepatectomy liver failure, and posthepatectomy hemorrhage were 
recorded in line with the definitions by the International Study Group of Liver Surgery (ISGLS) [22]. Postoperative medical complications included the frequency of PE, cardiac complications, deep vein thrombosis, and acute renal failure. In addition, the following variables and outcomes were considered in the present analysis: need for and duration of portal triad clamping, operating time, total blood loss, perioperative transfusion, postoperative hospital stay, interventional drainage, reoperation, and mortality within 90 day of surgery.

\section{Study interventions and perioperative care}

Hepatic resections were performed in a standardized fashion as outlined in the study publications [8, 15-18]. In brief, resections were carried out via a laparotomy under low CVP $(<5 \mathrm{mmHg})$ without routine use of vascular inflow control (only in case of significant intraoperative blood loss). Parenchymal transection was achieved by clamp-crushing technique, stapler, ultrasonic dissection, or a sealing energy device. Topical agents and argon beam coagulation were used at the discretion of the surgeon.

Except for the randomized trial on infrahepatic IVC clamping, clamping of the IVC was performed at discretion of the surgeon and anesthesiologist. It was carried out below the hepatoduodenal ligament and above the right renal vein using a vascular clamp. Initially, the infrahepatic IVC was clamped for a short period. In case the patient tolerated occlusion of the IVC, the vascular clamp was applied for complete clamping of the infrahepatic IVC for the entire period of hepatic parenchymal transection. Infrahepatic IVC clamping was applied on the study group only.

\section{Statistical analysis}

Categorical variables were summarized by absolute and relative frequencies (percentage) and compared using Pearson's $\chi^{2}$ or Fisher's exact test. Continuous variables were expressed as mean (standard deviation) or median (interquartile range) and compared with Student's $t$-test or Wilcoxon-Mann-Whitney test depending on the pattern of distribution. The Holm-Sidak method was used to adjust for multiple $t$-testing. A bivariate logistic regression analysis of variables $(P<0.05)$ was performed to determine risk factors of postoperative PE. A generic inverse-variance method was conducted using a fixed-effects and random effects model to assess differences between the individual studies for the rate of PE. Odds ratios (ORs) with 95\% CI were calculated for binary outcomes. The interstudy heterogeneity $\left(I^{2}\right)$ was assessed using the $I^{2}$ value. Subgroup analyses were performed for patients with major hepatectomy with and without vascular resections. A two- sided $P<0.05$ was deemed statistically significant. Statistical analysis was performed using $\mathrm{R}$ version 3.6.1.

\section{Results}

\section{Patient characteristics}

Of 594 patients who were included in prospective controlled trials, a total of 505 patients met the inclusion criteria. Of these, 141 patients received IVC clamping and 364 patients served as control group. The study flow diagram is shown in Fig. 1. Patients' baseline characteristics are outlined in Table 1. Patients in the IVC clamping group were younger $(59 \pm 12$ vs. $62 \pm 12, P=0.011)$ compared to the control group. Other baseline characteristics were similar in both groups.

\section{Operative details and intraoperative outcome}

The mean duration of IVC clamping in the clamping group was $17 \pm 16$ min with a total of 22 patients $(16 \%)$ having a clamping-time below $5 \mathrm{~min}$. Characteristics of surgery and intraoperative outcomes are summarized for both study groups in Table 2. CVP prior to resection was similar in both groups ( $5 \pm 3$ vs. $5 \pm 4 ; P=0.848)$. There were significantly more major liver resections (66\% vs. $50 \%$, $P=0.001)$ and a trend for more vascular resections $(9 \%$ vs. $6 \%, P=0.096$ ) in the IVC clamping group. As expected, the Pringle maneuver was applied more frequently in the IVC clamping group due to severe intraoperative bleeding, though this did not reach statistical significance (23\% vs. $19 \%, P=0.113$ ). Stapler and ultrasound-based devices were more frequently applied in the IVC clamping group compared to the control group, whereas more sealing devices were used for parenchymal dissection in the control group $(P<0.001)$. Despite a higher percentage of major hepatectomies and vascular resections in the IVC clamping group, there were no significant differences in intraoperative blood loss, operating time and the need for intraoperative blood transfusion between both groups.

\section{Incidence of postoperative PE}

Analysis of the primary endpoint revealed no significant difference in the rate of postoperative PE between the study groups ( $3 \%$ vs. $3 \%, P=0.762$ ). The pooled rate of $\mathrm{PE}$ in the individual studies using fixed and random effects models indicated the incidence of $\mathrm{PE}$ after infrahepatic IVC clamping to be limited to a single study only ( $P=0.564$ and $P=0.767$ ) (Fig. 2A). Next, further risk factors for the onset of PE were determined by univariate 
Fig. 1 Study flow diagram

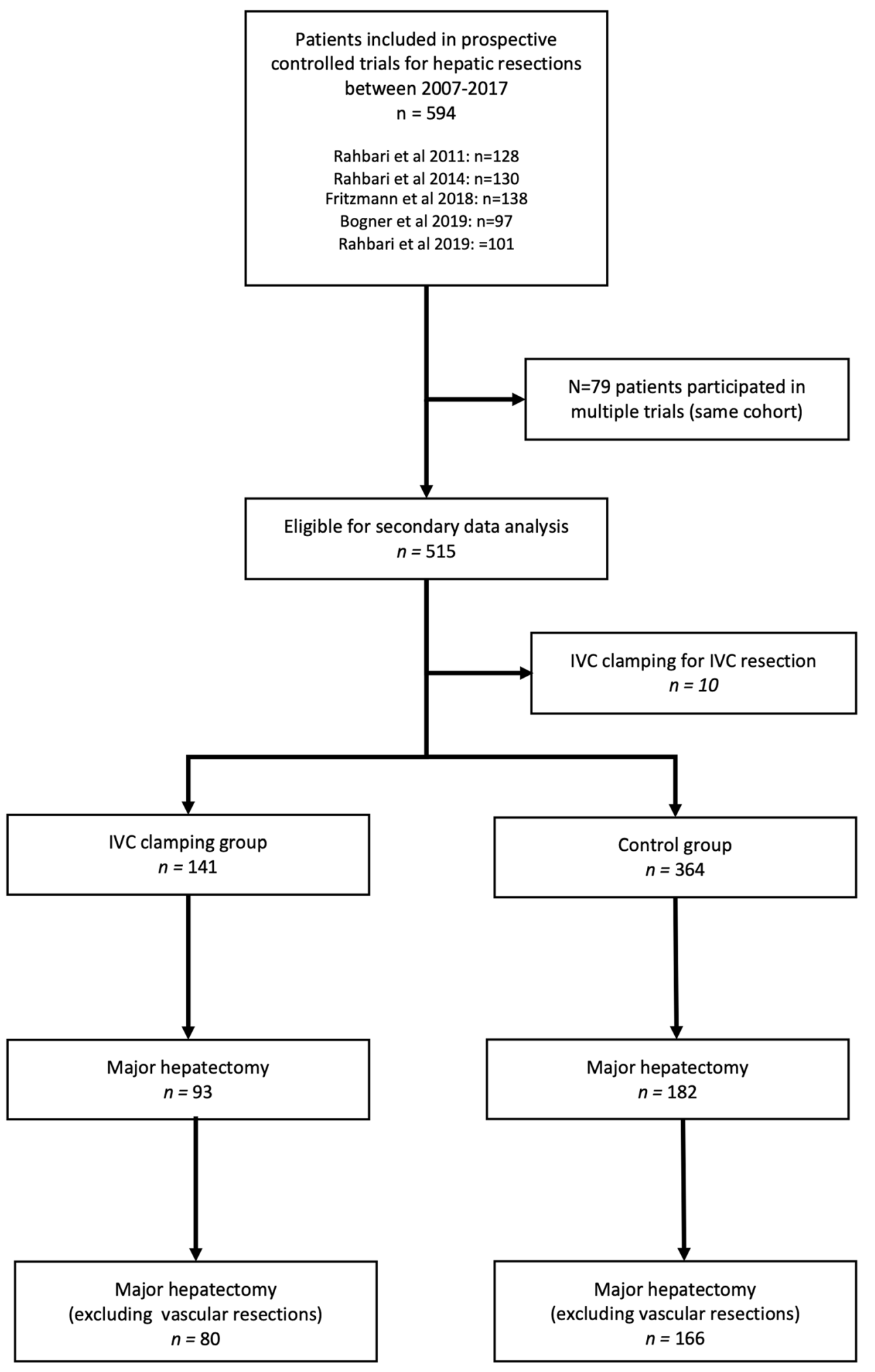

analysis. ASA $\geq 3(P=0.043)$ INR $<0.9(P=0.016)$, and intraoperative transfusion of fresh frozen plasma (FFP) $(P=0.017)$ were associated with postoperative PE. The total number of intraoperative transfused FFPs were comparable in patients with PE and without PE (4 (3-4) vs. $4(2-4), P=0.879)$. On multivariate analysis, none of these factors were independently associated with PE (Table 3). 
Table 1 Baseline characteristics

\begin{tabular}{|c|c|c|c|}
\hline & Infrahepatic IVC clamping $(n=141)$ & Control $(n=364)$ & $P$ value \\
\hline Age (years)* & $59(12)$ & $62(12)$ & 0.011 \\
\hline BMI $\left(\mathrm{kg} / \mathrm{m}^{2}\right)^{*}$ & $26(4)$ & $26(5)$ & 0.189 \\
\hline Sex ratio (male:female) & $80: 61$ & $222: 142$ & 0.382 \\
\hline ASA & & & 0.566 \\
\hline $\mathrm{I}$ & $4(3)$ & $7(2)$ & \\
\hline II & $63(45)$ & $142(38)$ & \\
\hline III & $74(52)$ & $212(58)$ & \\
\hline IV & 0 & $1(1)$ & \\
\hline Missing & - & $2(1)$ & \\
\hline Steatosis & & & 0.169 \\
\hline No & $50(35)$ & $98(27)$ & \\
\hline Grade $1+2$ & $84(60)$ & $249(68)$ & \\
\hline Grade $3+4$ & $5(4)$ & $15(4)$ & \\
\hline Missing & $2(1)$ & $2(1)$ & \\
\hline Fibrosis & & & 0.576 \\
\hline No & $67(48)$ & $186(51)$ & \\
\hline Grade $1+2$ & $65(46)$ & $155(42)$ & \\
\hline Grade 3 & $8(5)$ & $22(6)$ & \\
\hline Missing & $1(1)$ & $1(1)$ & \\
\hline Liver cirrhosis & & & 0.549 \\
\hline No & $128(91)$ & $314(86)$ & \\
\hline Child A & $12(8)$ & $45(12)$ & \\
\hline Child B & $1(1)$ & $5(2)$ & \\
\hline History of chemotherapy & $61(43)$ & $176(49)$ & 0.321 \\
\hline History of hepatic resection & $22(16)$ & $84(23)$ & 0.068 \\
\hline Diagnosis & & & 0.116 \\
\hline Primary liver malignancy & $47(33)$ & $114(31)$ & \\
\hline Secondary liver malignancy & $75(53)$ & $221(61)$ & \\
\hline Benign liver disease & $19(14)$ & $29(8)$ & \\
\hline \multicolumn{4}{|l|}{ Preoperative laboratory tests* } \\
\hline Bilirubin (mg/dl) & $0.8(1.4)$ & $0.8(1.8)$ & 0.828 \\
\hline $\mathrm{AP}(\mathrm{U} / \mathrm{l})$ & $164(161)$ & 147 (154) & 0.329 \\
\hline gGT (U/l) & $195(300)$ & $169(271)$ & 0.372 \\
\hline AST (U/l) & $38(39)$ & $39(34)$ & 0.801 \\
\hline ALT (U/l) & $44(51)$ & $42(46)$ & 0.627 \\
\hline Creatinine (mg/dl) & $0.8(0.2)$ & $0.8(0.3)$ & 0.293 \\
\hline Hemoglobin (g/dl) & $13(2)$ & $13(2)$ & 0.205 \\
\hline Platelets (/nl) & $275(120)$ & 275 (107) & 0.922 \\
\hline International normalized ratio & $1.0(0.1)$ & $1.0(0.1)$ & 0.232 \\
\hline
\end{tabular}

Values in parentheses are percentages unless indicated otherwise; ${ }^{\text {Values are mean (s.d.) }}$

$I V C$ inferior vena cava, ASA American Society of Anesthesiologists, BMI body mass index, $A P$ alkaline phosphatase, $g G T$ gamma glutamyl transferase, $A S T$ aspartate aminotransferase, $A L T$ alanine aminotransferase

Of note, the analysis of general and specific complications following hepatectomy, such as posthepatectomy bile leakage, posthepatectomy liver failure, and posthepatectomy hemorrhage revealed comparable rates between the study groups (Table 4). None of the deaths were attributed to PE. 


\section{Subgroup analysis of patients with major hepatectomy and vascular resections}

As major hepatic hepatectomies and vascular resections were more common in the IVC clamping group, we performed a subgroup analysis on the effects of infrahepatic IVC clamping on perioperative outcome of patients who underwent a major hepatectomy with or without vascular
Fig. 2 Forrest plot of the rate of postoperative pulmonary embolism The pooled rate of postoperative pulmonary embolism (PE) in the infrahepatic IVC clamping and control group is shown in the total cohort (a), in the major hepatectomy cohort (b), and in the major hepatectomy cohort without vascular resections (c). An inversevariance random effects model was used for pooling of the rate of PE in the studies. Squares and horizontal bars indicate point estimate (odds ratios) with $95 \% \mathrm{CI}$ for the individual studies

Table 2 Operative details and intraoperative outcome

\begin{tabular}{|c|c|c|c|}
\hline & Infrahepatic IVC clamping $(n=141)$ & Control $(n=364)$ & $P$ value \\
\hline CVP prior resection $*$ & $5(3)$ & $5(4)$ & 0.848 \\
\hline Extent of resection & & & 0.001 \\
\hline Major hepatectomy & $93(66)$ & $181(50)$ & \\
\hline Minor hepatectomy & $48(34)$ & $183(40)$ & \\
\hline Type of resection & & & 0.010 \\
\hline Right/extended right hemihepatectomy & $52(37)$ & $117(32)$ & \\
\hline Left/extended left hemihepatectomy & $37(26)$ & $56(15)$ & \\
\hline Central hepatectomy & $2(1)$ & $8(2)$ & \\
\hline Anatomic resection $>2$ segments & $4(4)$ & $2(1)$ & \\
\hline Anatomic resection $\leq 2$ segments & $29(20)$ & $87(24)$ & \\
\hline Non-anatomical resections & $17(12)$ & $94(26)$ & \\
\hline No. of resected segments $\dagger$ & $4(2-5)$ & $4(1-4)$ & 0.001 \\
\hline Extrahepatic resection & $17(13)$ & $47(13)$ & 1.000 \\
\hline Vascular resection & $13(9)$ & $18(5)$ & 0.096 \\
\hline Portal vein & $9(6)$ & $8(2)$ & \\
\hline Hepatic artery & $3(2)$ & $2(1)$ & \\
\hline Portal vein + hepatic artery & $1(1)$ & $2(1)$ & \\
\hline Hepatic vein & 0 & $6(1)$ & \\
\hline Bilioenteric anastomosis & $24(16)$ & $50(14)$ & 0.400 \\
\hline Resection device & & & $<0.001$ \\
\hline Crush clamp & $21(15)$ & $56(15)$ & \\
\hline Stapler & $78(55)$ & $159(43)$ & \\
\hline Ultrasound-based & $15(11)$ & $13(4)$ & \\
\hline Sealing device & 27 (19) & $134(37)$ & \\
\hline Missing & - & $2(1)$ & \\
\hline Pringle maneuver & $36(23)$ & 69 (19) & 0.113 \\
\hline Duration of pringle maneuver $(\mathrm{min})^{*}$ & $12(9)$ & $16(15)$ & 0.145 \\
\hline Operating time $(\min )^{*}$ & $205(100)$ & $210(102)$ & 0.685 \\
\hline Total blood loss $(\mathrm{ml}) \dagger$ & $800(450-1400)$ & $800(500-1400)$ & 0.679 \\
\hline Mean blood loss* & $1100(1000)$ & $1100(1000)$ & 0.956 \\
\hline Intraoperative transfusion & $25(18)$ & $59(16)$ & 0.274 \\
\hline PRBCs $\dagger$ & $2(2-4)$ & $2(2-4)$ & 0.500 \\
\hline $\mathrm{FFP} \dagger$ & $4(3-5)$ & $4(2-4)$ & 0.103 \\
\hline MABP prior resection* & 77 (13) & $76(13)$ & 0.323 \\
\hline SBP prior resection* & 108 (19) & $108(20)$ & 0.600 \\
\hline
\end{tabular}

Values in parentheses are percentages unless indicated otherwise; ${ }^{\dagger}$ Values are median (iqr); *Values are mean (s.d.)

$I V C$ inferior vena cava, $C V P$ central venous, $P R B C$ packed red blood cell, $F F P$ fresh frozen plasma, pressure. $M A B P$ mean arterial blood pressure, $S B P$ systolic blood pressure 


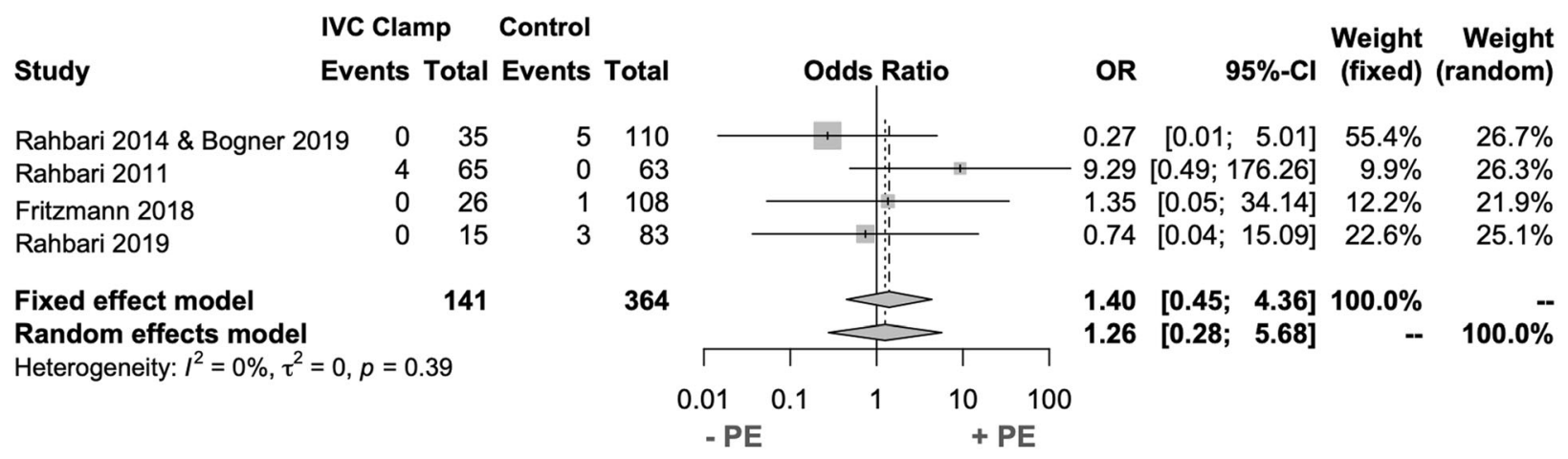

Test for overall effect (Fixed effect model): $Z=0.58(p=0.564)$

Test for overall effect (Random effects model): $Z=0.39(p=0.767)$

A Pooled rate of postoperative pulmonary embolism

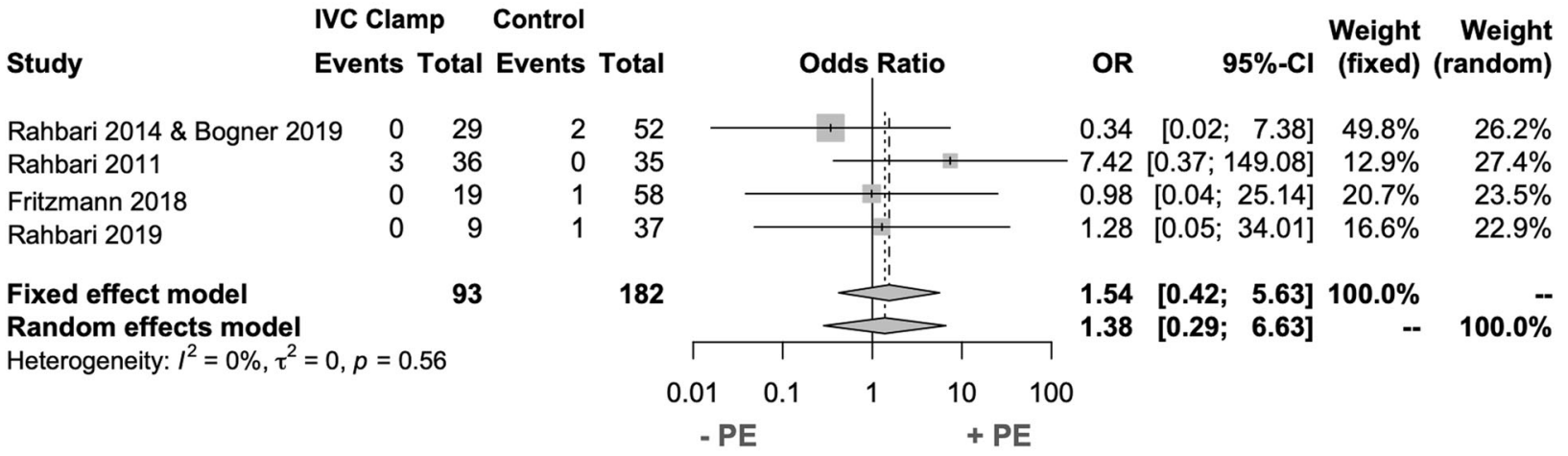

Test for overall effect (Fixed effect model): $Z=0.65(p=0.513)$

Test for overall effect (Random effects model): $Z=0.40(p=0.689)$

B Pooled rate of postoperative pulmonary embolism after major hepatectomy

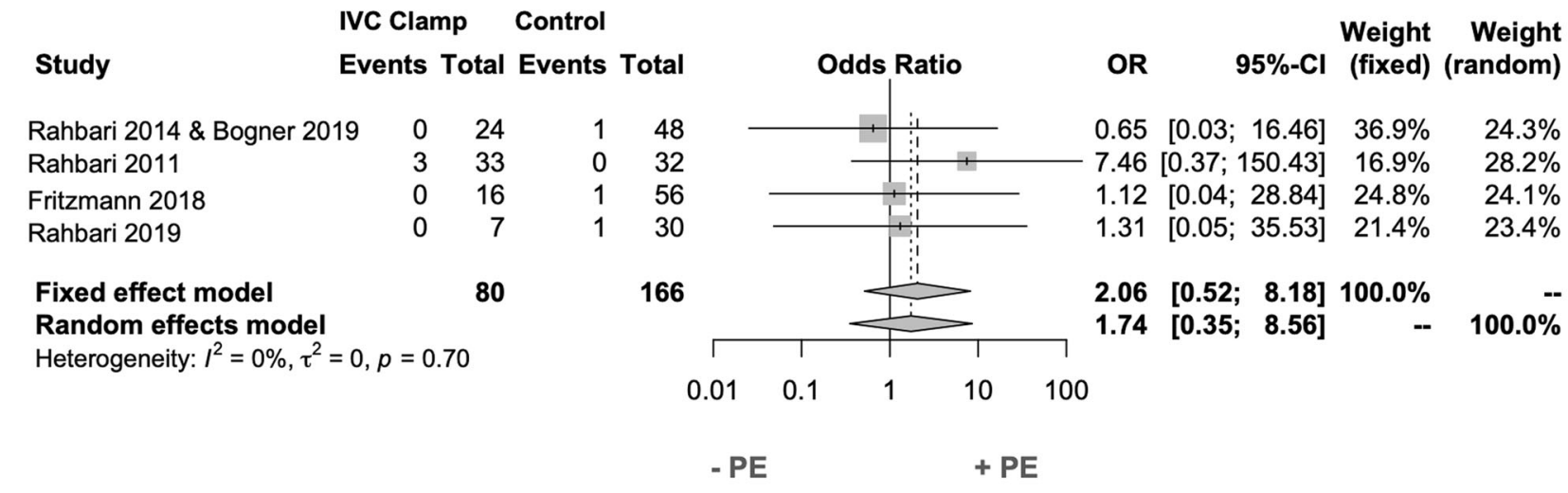

Test for overall effect (Fixed effect model): $Z=1.03(p=0.305)$

Test for overall effect (Random effects model): $Z=0.68(p=0.498)$

C Pooled rate of postoperative pulmonary embolism after major hepatectomy (excluding vascular resections) 
Table 3 Clinicopathological factors associated with postoperative pulmonary embolism

\begin{tabular}{|c|c|c|c|c|c|}
\hline & \multicolumn{3}{|l|}{ Univariate } & \multicolumn{2}{|l|}{ Multivariate } \\
\hline & $\mathrm{PE}(n=13)$ & No PE $(n=492)$ & $P$ value & OR $(95 \% \mathrm{CI})$ & $P$ value \\
\hline Age (years)* & $65(11)$ & $61(12)$ & 0.166 & & \\
\hline $\operatorname{BMI}\left(\mathrm{kg} / \mathrm{m}^{2}\right)^{*}$ & $27(3)$ & $26(5)$ & 0.419 & & \\
\hline Sex ratio (male:female) & $7: 6$ & $295: 197$ & 0.657 & & \\
\hline ASA $\geq$ III & $11(85)$ & $278(57)$ & 0.043 & $3.09(0.64-14.99)$ & 0.162 \\
\hline Steatosis grade $\geq 3$ & 0 & $24(5)$ & 0.412 & & \\
\hline Fibrosis grade $\geq 3$ & $1(8)$ & $29(6)$ & 0.790 & & \\
\hline Liver cirrhosis & $2(15)$ & $61(12)$ & 0.748 & & \\
\hline Primary or secondary liver malignancies & $10(77)$ & $447(91)$ & 0.091 & & \\
\hline History of hepatic resections & $2(15)$ & $104(21)$ & 0.615 & & \\
\hline History of chemotherapy & $6(46)$ & $231(47)$ & 0.955 & & \\
\hline Platelets $\geq 450 / \mathrm{nl}$ & 0 & $36(8)$ & 0.301 & & \\
\hline INR $<0.9$ & $1(8)$ & $4(1)$ & 0.016 & NA & \\
\hline \multicolumn{6}{|l|}{ Preoperative laboratory tests } \\
\hline Bilirubin (mg/dl) & $0.6(0.4)$ & $0.8(1.6)$ & 0.597 & & \\
\hline Creatinine (mg/dl) & $0.8(0.1)$ & $0.9(0.3)$ & 0.326 & & \\
\hline AP (U/l) & $147(135)$ & $152(156)$ & 0.914 & & \\
\hline gGT (U/l) & $204(221)$ & $175(281)$ & 0.713 & & \\
\hline AST (U/l) & $38(34)$ & $39(36)$ & 0.926 & & \\
\hline $\operatorname{ALT}(\mathrm{U} / \mathrm{l})$ & $38(25)$ & $43(49)$ & 0.690 & & \\
\hline Hemoglobin (g/dl) & $13(2)$ & $13(2)$ & 0.825 & & \\
\hline CVP prior resection $*$ & $6(4)$ & $5(4)$ & 0.611 & & \\
\hline Major hepatectomy & $7(54)$ & $267(54)$ & 0.976 & & \\
\hline No. of resected segments $\dagger$ & $4(4-5)$ & $4(4-5)$ & 0.675 & & \\
\hline Vascular resection & $1(8)$ & $30(6)$ & 0.813 & & \\
\hline Extrahepatic resection & $2(15)$ & $62(13)$ & 0.784 & & \\
\hline Bilioenteric anastomosis & $4(31)$ & $70(14)$ & 0.096 & & \\
\hline IVC clamping & $4(31)$ & $137(28)$ & 0.817 & $1.55(0.42-5.70)$ & 0.506 \\
\hline \multicolumn{6}{|l|}{ Intraoperative transfusions } \\
\hline PRBC & $2(15)$ & $82(17)$ & 0.902 & & \\
\hline FFP & $3(30)$ & $33(8)$ & 0.017 & $3.99(0.96-16.23)$ & 0.055 \\
\hline Pringle maneuver & 0 & $105(21)$ & 0.061 & & \\
\hline Operating time $(\min )^{*}$ & $226(70)$ & $206(100)$ & 0.481 & & \\
\hline Total blood loss $(\mathrm{ml}) \dagger$ & $1000(700-1400)$ & $800(400-1400)$ & 0.203 & & \\
\hline Mean blood loss $(\mathrm{ml})^{*}$ & $1200(700)$ & $1000(1000)$ & 0.750 & & \\
\hline MABP prior resection* & $69(8)$ & $76(13)$ & 0.132 & & \\
\hline SBP prior resection* & $106(21)$ & $109(19)$ & 0.570 & & \\
\hline
\end{tabular}

Values in parentheses are percentages unless indicated otherwise; ${ }^{\dagger}$ Values are median (iqr); *Values are mean s.d.);

$I V C$ inferior vena cava, $P E$ pulmonary embolism, $A S A$ American Society of Anesthesiologists, BMI body mass index, INR International normalized ratio, $A S T$ aspartate aminotransferase, $A L T$ alanine aminotransferase, $I V C$ inferior vena cava, $P R B C$ packed red blood cell, $F F P$ fresh frozen plasma, $M A B P$ mean arterial blood pressure, $S B P$ systolic blood pressure, $C V P$ central venous pressure, $O R$ odds ratio, $C I$ confidence interval

resections (Table 5). Again, there was no significant difference in the incidence of postoperative PE between major hepatectomy patients with and without IVC clamping in the vascular resection ( $3 \%$ vs. $2 \%, P=0.692)$ and no vascular resection groups $(3 \%$ vs. $2 \%, P=0.394)$. In line with these data, the pooled rate of $\mathrm{PE}$ was comparable in the individual studies (OR 1.26, 95\%CI 0.45-4.36, $P=0.767)$ as well as in the subgroups with major 
Table 4 Postoperative outcome

\begin{tabular}{|c|c|c|c|}
\hline & Infrahepatic IVC clamping $(n=141)$ & Control $(n=364)$ & $P$ value \\
\hline Pulmonary embolism & $4(3)$ & $9(3)$ & 0.762 \\
\hline Cardiac complication & $6(4)$ & $19(5)$ & 0.820 \\
\hline Deep vein thrombosis & $3(2)$ & $14(4)$ & 0.420 \\
\hline Acute renal failure & $3(2)$ & $16(4)$ & 0.302 \\
\hline Abdominal fluid collection & $17(12)$ & $41(11)$ & 0.876 \\
\hline Postoperative transfusion & $16(13)$ & $35(9)$ & 0.274 \\
\hline $\mathrm{PRBCs} \dagger$ & $2(2)$ & $2(2-3)$ & 0.908 \\
\hline $\mathrm{FFP} \dagger$ & $4(2)$ & $4(2-7)$ & 0.690 \\
\hline Bile leakage & $34(24)$ & $71(20)$ & 0.272 \\
\hline Grade B/C & $25(18)$ & $55(15)$ & 0.378 \\
\hline Posthepatectomy hemorrhage & $8(6)$ & $9(3)$ & 0.095 \\
\hline Grade B/C & $5(4)$ & $7(2)$ & 0.397 \\
\hline Posthepatectomy liver failure & $14(10)$ & $31(8)$ & 0.605 \\
\hline Grade B/C & $11(8)$ & $25(7)$ & 0.541 \\
\hline Radiological intervention & $35(25)$ & $94(26)$ & 0.910 \\
\hline Reoperation & $19(14)$ & $39(11)$ & 0.437 \\
\hline Clavien-Dindo classification & & & 0.932 \\
\hline Grade I & $8(5)$ & $33(9)$ & \\
\hline Grade II & $20(14)$ & $51(14)$ & \\
\hline Grade III & $39(28)$ & $100(28)$ & \\
\hline Grade IV & $7(5)$ & $19(6)$ & \\
\hline Grade V (death) & $9(6)$ & $24(6)$ & 0.941 \\
\hline Length of postoperative stay (days) $\dagger$ & $11(8-19)$ & $11(7-20)$ & 0.852 \\
\hline
\end{tabular}

Values in parentheses are percentages unless indicated otherwise; ${ }^{\dagger}$ Values are median (iqr); *Values are mean (s.d.)

$I V C$ inferior vena cava, $P R B C$ packed red blood cell, $F F P$ fresh frozen plasma

hepatectomy and vascular resection (OR 1.54, 95\% CI $0.42-5.63, P=0.689)$ or no vascular resection (OR 1.74, 95\%CI 0.35-8.56, $P=0.498$ ), respectively (Fig. $2 \mathrm{~B}, \mathrm{C}$ ). Intraoperative blood loss was reduced following IVC clamping in both subgroups, though these differences failed to reach statistical significance $(P=0.197$ and $P=0.128)$. Other intraoperative outcomes were well-balanced between the subgroups. Furthermore, the number of patients with general postoperative complications and specific complications following hepatectomy was wellbalanced.

\section{Discussion}

Infrahepatic IVC clamping to reduce blood loss was first introduced in 2004 by Otsubo et al. [6] Although this technique was shown to be safe in various cohort studies $[23,24]$, serious concerns of postoperative PE were raised after the results of a randomized controlled trial were published [8]. Five other randomized trials evaluated the impact of infrahepatic IVC clamping on intraoperative blood loss; however, none but two of these studies outlined postoperative complications in detail [9-12, 19]. Chen et al. included a selective cohort of cirrhotic patients with HCC who underwent mesohepatectomies and detected comparable morbidity rates in infectious complications, pleural effusion, ascites, and hepatic encephalopathy, respectively [19]. Ueno et al. applied a different IVC clamping technique (partial clamping) and reported exclusively on liver-specific complications (e.g., bile leak, pleural-effusion ascites, hyperbilirubinemia) in a majority of patients with primary liver malignancies (79\%) and minor hepatectomies (53\%) [12]. Therefore, two recent meta-analyses comprising six randomized trials with heterogeneous surgical techniques, perioperative care, and inclusion of selective patient cohorts, failed to give conclusive evidence on this topic [13, 14]. Moreover, Fancellu et al. raised serious concerns in their meta-analysis about a potential type II-error with regard to fewer complications after IVC clamping [14]. The present study addressed this lack of evidence by secondary data analysis of prospective 
Table 5 Subgroup analysis of perioperative outcomes in patients with major hepatic resections

\begin{tabular}{|c|c|c|c|c|c|c|}
\hline & \multicolumn{3}{|l|}{ Vascular resection } & \multicolumn{3}{|l|}{ No vascular resection } \\
\hline & $\begin{array}{l}\text { Infrahepatic IVC } \\
\text { clamping }(n=80)\end{array}$ & $\begin{array}{l}\text { Control } \\
(n=166)\end{array}$ & $P$ value & $\begin{array}{l}\text { Infrahepatic IVC } \\
\text { clamping }(n=93)\end{array}$ & $\begin{array}{l}\text { Control } \\
(n=182)\end{array}$ & $P$ value \\
\hline \multicolumn{7}{|l|}{ Intraoperative outcome } \\
\hline CVP prior to resection $*$ & $5(3)$ & $5(3)$ & 0.167 & $5(3)$ & $5(3)$ & 0.197 \\
\hline Extrahepatic resection & $16(17)$ & $24(13)$ & 0.333 & $12(15)$ & $18(11)$ & 0.314 \\
\hline Bilioenteric anastomosis & $23(25)$ & $47(25)$ & 0.885 & $11(14)$ & $37(22)$ & 0.125 \\
\hline Pringle maneuver & $23(25)$ & $43(24)$ & 0.839 & $20(12)$ & $38(23)$ & 0.715 \\
\hline Operating time $(\min ) *$ & $230(106)$ & $240(107)$ & 0.443 & $206(81)$ & $229(97)$ & 0.067 \\
\hline Total blood loss $(\mathrm{ml}) \dagger$ & $1000(500-1450)$ & $1000(650-1800)$ & 0.197 & $900(500-1400)$ & $1000(700-1700)$ & 0.128 \\
\hline Mean blood loss & $1200(1100)$ & $1400(1300)$ & 0.336 & $1200(1200)$ & $1400(1300)$ & 0.515 \\
\hline Intraoperative transfusion & $20(22)$ & $42(23)$ & 0.879 & $17(21)$ & $35(21)$ & 1.000 \\
\hline PRBCs $\dagger$ & $3(2-4)$ & $2(2-4)$ & 0.709 & $3(2-4)$ & $2(2-4)$ & 0.330 \\
\hline $\mathrm{FFP} \dagger$ & $4(3-5)$ & $4(2-4)$ & 0.530 & $4(3-4)$ & $4(2-4)$ & 0.372 \\
\hline MABP prior resection * & $77(14)$ & $75(13)$ & 0.589 & $76(13)$ & $76(13)$ & 0.451 \\
\hline SBP prior resection $*$ & $108(19)$ & $110(23)$ & 0.561 & $109(17)$ & $109(23)$ & 0.954 \\
\hline \multicolumn{7}{|l|}{ Postoperative outcome } \\
\hline Pulmonary embolism & $3(3)$ & $4(2)$ & 0.692 & $3(4)$ & $3(2)$ & 0.394 \\
\hline Cardiac complication & $6(7)$ & $11(6)$ & 1.000 & $6(8)$ & $9(5)$ & 0.573 \\
\hline Deep vein thrombosis & $3(3)$ & $8(4)$ & 0.755 & $2(3)$ & $6(4)$ & 1.000 \\
\hline Acute renal failure & $3(3)$ & $11(6)$ & 0.395 & $2(3)$ & $8(5)$ & 1.000 \\
\hline Abdominal fluid collection & $12(13)$ & $27(15)$ & 0.718 & $9(11)$ & $22(13)$ & 0.838 \\
\hline Postoperative transfusion & $17(18)$ & $23(13)$ & 0.189 & $12(15)$ & $20(12)$ & 0.548 \\
\hline Bile leakage & $30(32)$ & $50(28)$ & 0.483 & $27(34)$ & $45(27)$ & 0.298 \\
\hline Grade $\mathrm{B} / \mathrm{C}$ & $22(25)$ & $43(24)$ & 0.280 & $20(25)$ & $38(23)$ & 0.348 \\
\hline $\begin{array}{l}\text { Posthepatectomy } \\
\text { hemorrhage }\end{array}$ & $7(8)$ & $5(3)$ & 0.078 & $7(9)$ & $4(3)$ & 0.042 \\
\hline Grade $\mathrm{B} / \mathrm{C}$ & $4(3)$ & $6(3)$ & 0.106 & $4(6)$ & $4(2)$ & 0.283 \\
\hline $\begin{array}{l}\text { Posthepatectomy liver } \\
\text { failure }\end{array}$ & $14(14)$ & $26(13)$ & 0.858 & $10(13)$ & $21(13)$ & 1.000 \\
\hline Grade $\mathrm{B} / \mathrm{C}$ & $11(11)$ & $21(11)$ & 0.751 & $8(10)$ & $16(9)$ & 0.678 \\
\hline Radiological intervention & $28(30)$ & $66(36)$ & 0.348 & $22(28)$ & $52(31)$ & 0.557 \\
\hline Reoperation & 18 (19) & $27(15)$ & 0.389 & $14(18)$ & $21(13)$ & 0.333 \\
\hline $\begin{array}{r}\text { Clavien-Dindo } \\
\text { classification }\end{array}$ & & & 0.852 & & & 0.713 \\
\hline Grad I & $6(6)$ & $16(9)$ & & $6(7)$ & $16(10)$ & \\
\hline Grade II & $13(14)$ & $27(15)$ & & $9(12)$ & $26(16)$ & \\
\hline Grade III & $28(30)$ & $62(34)$ & & $25(31)$ & $54(32)$ & \\
\hline Grade IV & $6(7)$ & $12(7)$ & & $4(5)$ & $10(6)$ & \\
\hline Grade V (death) & $9(10)$ & $21(11)$ & 0.828 & $6(8)$ & $17(10)$ & 0.789 \\
\hline $\begin{array}{l}\text { Length of postoperative } \\
\text { stay (days) } \dagger\end{array}$ & $15(9-23)$ & $15(9-28)$ & 0.501 & $12(9-22)$ & $15(8-25)$ & 0.520 \\
\hline
\end{tabular}

Values in parentheses are percentages unless indicated otherwise; †Values are median (iqr); *Values are mean (s.d.)

$C V P$ central venous pressure, $M A B P$ mean arterial blood pressure, $S B P$ systolic blood pressure

trials including patients who underwent hepatic resections with standardized surgical techniques and perioperative management. The results showed that infrahepatic IVC clamping was neither associated with $\mathrm{PE}$, nor with other postoperative complications. A further subgroup analysis 
of patients who underwent major hepatic resection with and without vascular resection confirmed these findings.

In previous studies, the onset of PE after liver resection was associated with high BMI, major hepatectomy, liver fibrosis, and previous thromboembolic events [25, 26]. While we detected no independent risk factors for PE, we observed a rather low overall incidence of PE after hepatic resection in our prospectively acquired dataset. While the incidence of PE was 3\% in our patients, an incidence of $6 \%$ is reported for patients undergoing hepatic resection in the literature [25, 27]. Still, less than half of our patients with PE had peripheral thrombosis suggesting thromboembolic events during liver resection and/or regeneration with accompanying activation of the coagulation cascade [28]. Historically, the risk of venous thromboembolism after hepatectomy was considered to be rare due to decreased postoperative synthesis of clotting factors [29]. However, there is rising evidence of hypercoagulability after hepatobiliary surgery advocating routine perioperative thromboprophylaxis [30]. In the present analysis, all included patients underwent routine thromboprophylaxis. Of note, the rate of clinically relevant posthepatectomy hemorrhage was found to be comparable to other studies [31, 32].

Compared to our previous trial on infrahepatic IVC clamping, we could not detect a significant decrease of total blood loss in patients with infrahepatic IVC clamping [8]. Although two included trials (NCT01858987, NCT00732979) clearly showed a benefit of IVC clamping, in particular, in patients with major hepatectomy, the difference of blood loss was subtle in the other studies resulting in a balanced amount of blood loss between the study groups. However, IVC clamping was performed in four out of five studies irrespective of the CVP prior to resection and on behalf of the surgeons which might have caused heterogenous results. We and other groups previously showed that there is no correlation between CVP and intraoperative blood loss in the range of low CVP values $[8,33]$. The Pringle maneuver was only used in $20 \%$ of the included patients and limited to cases with significant blood loss. IVC clamping was well-tolerated in the patient cohort with $>84 \%$ of the patients having a clampingtime $>5 \mathrm{~min}$. Unfortunately, we could not assess blood loss during hepatic transection in the patient cohort, which might have been the more suitable outcome parameter addressing this question. Further, the study and control groups had significant discrepancies regarding the extent of hepatectomy and vascular resections. Although we performed subgroup analyses to adjust the study and control groups, the effect of IVC clamping could have been reduced due to other non-balanced unknown factors.

There are some limitations to the present study. First, this was a secondary data analysis of available data from prospective trials of our study group prohibiting definitive conclusions. In fact, only a few trials addressed the impact of IVC clamping in liver surgery so far and, in particular, there is lacking data in patients with secondary liver malignancies. Therefore, large-scale multi-institutional studies are needed to provide conclusive results. Second, we assessed the impact of IVC clamping on a selected patient cohort without severe comorbidities, advanced liver cirrhosis, or known coagulopathies. This might have caused some selection bias. Third, all patients underwent conventional hepatic resection. As minimally invasive surgery is an emerging field in liver surgery, the results of the present study might not be transferable to laparoscopic resections because pneumoperitoneum and patient positioning may result in unreliable CVP values [34, 35]. Although a recent randomized trial demonstrated that lowering of CVP values in laparoscopic hepatectomy was associated with lower blood loss, IVC clamping was omitted in this trial and the safety and impact in minimally invasive surgery remains unclear [36]. Fourth, we did not include studies with the restricted use of, e.g., anteriorapproach hepatectomy for large primary liver malignancies. Therefore, our findings should be interpreted with caution in other patient cohorts.

\section{Conclusion}

We found no adverse impact of IVC clamping during conventional hepatic resection. In particular, there was no association with $\mathrm{PE}$ after elective hepatic resection in the present study cohort. Infrahepatic IVC clamping may therefore be applied as a safe technique to reduce CVP in patients undergoing elective hepatic resection.

Acknowledgements The authors report no proprietary or commercial interest in any product mentioned or concept discussed in this article.

Author contributions EB: conceptualization, methodology, writingoriginal draft preparation, investigation, visualization; Arianeb Mehrabi, Dorothée Sturm, Christoph Reissfelder, JW: data curation, investigation, writing-reviewing and editing; NNR: conceptualization, methodology, writing-reviewing and editing, supervision, investigation. All of the authors have read and approved the manuscript.

Funding Open Access funding enabled and organized by Projekt DEAL.

\section{Declarations}

Conflict of interest The authors declare no conflict of interest.

Open Access This article is licensed under a Creative Commons Attribution 4.0 International License, which permits use, sharing, adaptation, distribution and reproduction in any medium or format, as long as you give appropriate credit to the original author(s) and the 
source, provide a link to the Creative Commons licence, and indicate if changes were made. The images or other third party material in this article are included in the article's Creative Commons licence, unless indicated otherwise in a credit line to the material. If material is not included in the article's Creative Commons licence and your intended use is not permitted by statutory regulation or exceeds the permitted use, you will need to obtain permission directly from the copyright holder. To view a copy of this licence, visit http://creativecommons. org/licenses/by/4.0/.

\section{References}

1. Poon RT, Fan ST, Lo CM et al (2004) Improving perioperative outcome expands the role of hepatectomy in management of benign and malignant hepatobiliary diseases: analysis of 1222 consecutive patients from a prospective database. Ann Surg 240(4):698-708

2. Birgin E, Rasbach E, Reissfelder C et al (2020) A systematic review and meta-analysis of caudate lobectomy for treatment of hilar cholangiocarcinoma. Eur J Surg Oncol 46(5):747-753

3. Jarnagin WR, Gonen M, Fong $Y$ et al (2002) Improvement in perioperative outcome after hepatic resection: analysis of 1,803 consecutive cases over the past decade. Ann Surg 236(4):397-406

4. Cescon M, Vetrone G, Grazi GL et al (2009) Trends in perioperative outcome after hepatic resection: analysis of 1500 consecutive unselected cases over 20 years. Ann Surg 249(6):995-1002

5. Jones RM, Moulton CE, Hardy KJ (1998) Central venous pressure and its effect on blood loss during liver resection. Br J Surg 85(8):1058-1060

6. Otsubo T, Takasaki K, Yamamoto M et al (2004) Bleeding during hepatectomy can be reduced by clamping the inferior vena cava below the liver. Surgery 135(1):67-73

7. Rahbari NN, Zimmermann JB, Koch $M$ et al (2009) IVC CLAMP: infrahepatic inferior vena cava clamping during hepatectomy-a randomised controlled trial in an interdisciplinary setting. Trials 10:94

8. Rahbari NN, Koch M, Zimmermann JB et al (2011) Infrahepatic inferior vena cava clamping for reduction of central venous pressure and blood loss during hepatic resection: a randomized controlled trial. Ann Surg 253(6):1102-1110

9. Kato M, Kubota K, Kita J et al (2008) Effect of infra-hepatic inferior vena cava clamping on bleeding during hepatic dissection: a prospective, randomized, controlled study. World J Surg 32(6):1082-1087. https://doi.org/10.1007/s00268-007-9445-0

10. Zhu P, Lau WY, Chen YF et al (2012) Randomized clinical trial comparing infrahepatic inferior vena cava clamping with low central venous pressure in complex liver resections involving the Pringle manoeuvre. Br J Surg 99(6):781-788

11. Zhou YM, Sui CJ, Zhang XF et al (2016) Anterior approach combined with infrahepatic inferior vena cava clamping right hepatic resection for large hepatocellular carcinoma: a prospective randomized controlled trial. Medicine (Baltimore) 95(27):e4159

12. Ueno M, Kawai M, Hayami S et al (2017) Partial clamping of the infrahepatic inferior vena cava for blood loss reduction during anatomic liver resection: a prospective, randomized, controlled trial. Surgery 161(6):1502-1513

13. Zhou Y, Zhang Z, Wan T (2018) Effect of infrahepatic inferior vena cava clamping on bleeding during hepatic resection: a metaanalysis. Asian J Surg 41(6):523-529

14. Fancellu A, Petrucciani N, Melis M et al (2018) Usefulness of infra-hepatic inferior vena cava clamping during liver resection: a meta-analysis of randomized controlled trials. J Gastrointest Surg 22(5):941-951

15. Rahbari NN, Elbers H, Koch M et al (2014) Randomized clinical trial of stapler versus clamp-crushing transection in elective liver resection. Br J Surg 101(3):200-207

16. Fritzmann J, Kirchberg J, Sturm D et al (2018) Randomized clinical trial of stapler hepatectomy versus ligasure transection in elective hepatic resection. Br J Surg 105(9):1119-1127

17. Bogner A, Reissfelder C, Striebel F et al (2019) Intraoperative increase of portal venous pressure is an immediate predictor of posthepatectomy liver failure after major hepatectomy: a prospective study. Ann Surg. https://doi.org/10.1097/SLA.0000000000003496

18. Rahbari NN, Birgin E, Sturm D et al (2019) Randomized clinical trial of BioFoam $(\mathrm{R})$ surgical matrix to achieve hemostasis after liver resection. HPB. https://doi.org/10.1016/j.hpb.2019.10.1529

19. Chen XP, Zhang ZW, Zhang BX et al (2006) Modified technique of hepatic vascular exclusion: effect on blood loss during complex mesohepatectomy in hepatocellular carcinoma patients with cirrhosis. Langenbecks Arch Surg 391(3):209-215

20. Goldhaber SZ (1998) Pulmonary embolism. N Engl J Med 339(2):93-104

21. Encke A, Haas S, Kopp I (2016) The prophylaxis of venous thromboembolism. Dtsch Arztebl Int 113(31-32):532-538

22. Koch M, Garden OJ, Padbury R et al (2011) Bile leakage after hepatobiliary and pancreatic surgery: a definition and grading of severity by the international study group of liver surgery. Surgery 149(5):680-688

23. Uchiyama K, Ueno M, Ozawa S et al (2009) Half clamping of the infrahepatic inferior vena cava reduces bleeding during a hepatectomy by decreasing the central venous pressure. Langenbecks Arch Surg 394(2):243-247

24. Imamura T, Yamamoto Y, Sugiura T et al (2019) Infrahepatic inferior vena cava semi-clamping can reduce blood loss during hepatic resection but still requires monitoring to avoid acute kidney injury. World J Surg 43(8):2038-2047. https://doi.org/10. 1007/s00268-019-04992-2

25. Melloul E, Dondero F, Vilgrain V et al (2012) Pulmonary embolism after elective liver resection: a prospective analysis of risk factors. J Hepatol 57(6):1268-1275

26. Nobili C, Marzano E, Oussoultzoglou E et al (2012) Multivariate analysis of risk factors for pulmonary complications after hepatic resection. Ann Surg 255(3):540-550

27. Reddy SK, Turley RS, Barbas AS et al (2011) Post-operative pharmacologic thromboprophylaxis after major hepatectomy: Does peripheral venous thromboembolism prevention outweigh bleeding risks? J Gastrointest Surg 15(9):1602-1610

28. Anderson FA Jr, Spencer FA (2003) Risk factors for venous thromboembolism. Circulation 107(23 Suppl 1):I9-16

29. De Pietri L, Montalti R, Begliomini B et al (2010) Thromboelastographic changes in liver and pancreatic cancer surgery: Hypercoagulability, hypocoagulability or normocoagulability? Eur J Anaesthesiol 27(7):608-616

30. Le AT, Harris JW, Maynard E et al (2017) Thromboelastography demonstrates perioperative hypercoagulability in hepato-pancreato-biliary patients and supports routine administration of preoperative and early postoperative venous thromboembolism chemoprophylaxis. HPB (Oxford) 19(2):154-161

31. Birgin E, Tesfazgi W, Knoth M et al (2019) Evaluation of the new ISGLS definitions of typical posthepatectomy complications. Scand J Surg 108(2):130-136

32. Rahbari NN, Birgin E, Bork U et al (2020) Anterior approach versus conventional hepatectomy for resection of colorectal liver metastasis: a randomized clinical trial. JAMA Surg. https://doi. org/10.1001/jamasurg.2020.5050 
33. Kim YK, Chin JH, Kang SJ et al (2009) Association between central venous pressure and blood loss during hepatic resection in 984 living donors. Acta Anaesthesiol Scand 53(5):601-606

34. Zhang W, Wang J, Li C et al (2017) Infrahepatic inferior vena cava clamping with pringle maneuvers for laparoscopic extracapsular enucleation of giant liver hemangiomas. Surg Endosc 31(9):3628-3636

35. Takahara T, Wakabayashi G, Konno H et al (2016) Comparison of laparoscopic major hepatectomy with propensity score matched open cases from the national clinical database in Japan. J Hepatobiliary Pancreat Sci 23(11):721-734

36. Pan YX, Wang JC, Lu XY et al (2020) Intention to control low central venous pressure reduced blood loss during laparoscopic hepatectomy: a double-blind randomized clinical trial. Surgery 167(6):933-941

Publisher's Note Springer Nature remains neutral with regard to jurisdictional claims in published maps and institutional affiliations. 